\title{
Passing the baton to pharmacists and nurses: New models of antibiotic stewardship for South Africa?
}

The decrease in effectiveness of antibiotics due to their overuse and misuse, resulting in increasing bacterial resistance, is a public health crisis of international concern, threatening modern medicine, animal health and food security. ${ }^{[1,2]}$ The recent identification of two new plasmid-mediated genes $(m c r-1 \text { and } m c r-2)^{[3,4]}$ that confer resistance to colistin, an antibiotic of last resort in the treatment of multidrugresistant Gram-negative bacteria, underscores a crisis that it is estimated will cause 10 million deaths per annum by 2050 , result in huge morbidity, and wipe out in excess of USD100 trillion from the world's economy. An international One Health response is required, co-ordinated at the highest level. ${ }^{[5]}$

South Africa (SA) is not exempt from this crisis, ${ }^{[6-8]}$ and has reacted by developing a national antimicrobial resistance strategy framework $^{[9]}$ and an implementation plan, ${ }^{[10]}$ interwoven with the government's annual performance plan. The One Health national response focuses on increasing public awareness, improving infection prevention, heightening surveillance of antibiotic consumption and resistance, and antibiotic stewardship (AS), the optimisation of appropriate antibiotic use. A practical guide to AS for SA is being developed to tackle the virtual absence of stewardship in hospitals and the community for antibiotics used in human and animal health. ${ }^{[1,12]}$

Such a guide will need to take into account what the best model for AS in SA would be. Human health AS programmes (ASPs) reported from resource-rich countries have shown that interventions to reduce excessive antibiotic prescribing can reduce resistance and improve microbiological and clinical outcomes, ${ }^{[13]}$ but do ASP models from high-income countries translate to SA? Historically, notable barriers to implementation of ASPs in SA have been a lack of healthcare professionals with the expertise to lead programmes, a problem compounded by geographical disparity, and a lack of understanding of the contextual and behavioural determinants that influence local prescribing practices.

The first ASPs introduced in SA followed the model of AS teams led by infection specialists at public sector, central academic hospitals and their outreach sites, which focused on AS ward rounds to change prescribing practice. ${ }^{[14]}$ The attractiveness of this model is its ability to reduce overall antibiotic use, prevent patients who do not require antibiotics from receiving them in the first place, and transfer AS skills to junior and senior doctors, microbiologists, pharmacists, nurses, infection control practitioners, medical students and other allied health professionals who attend the stewardship ward rounds. Two national training centres have now been funded to rapidly train prescriber-pharmacist-hospital manager teams from provincial hospitals throughout SA to promote the initiation of ASPs in their hospitals and in turn train local practitioners in stewardship.

More recently, a model focused on non-specialist pharmacists, which demonstrated a sustainable reduction in antibiotic use across 47 Netcare hospitals, has highlighted the opportunity to widen the net of interventionists. ${ }^{[15]}$ The Netcare model promoted collaborative action utilising existing resources and the concept of low-hanging fruit' across a diverse group of urban and rural SA private hospitals. The 5-year study, involving stepwise change management and quality improvement principles including behaviour change techniques, utilised non-specialised pharmacists to co-ordinate a prospective audit and feedback strategy targeting antibiotics used for $>7$ days, concurrent use of more than four antibiotics, redundant or double antibiotic coverage and increased use of microbiological cultures. Notably, the process measures were introduced following extensive consultation with all stakeholders, emphasising the need for change and establishing clinician collaboration in so doing.

The results were impressive: 116662 patients received antibiotics during 104 weeks of standardised audit, measurement and feedback, with 7934 interventions by pharmacists recorded for the targeted measures, indicating that almost one in 15 inpatient prescriptions required intervention. In a previous survey in SA intensive care units, ${ }^{[1]}$ antibiotic duration was found to be inappropriate in $53.2 \%$ and $81.7 \%$ of public and private sector patients, respectively. It was therefore not surprising that excessive duration represented the majority $(39.2 \%)$ of the pharmacist interventions. This simple protocol led to a reduction in mean antibiotic use, recorded as daily doses per 100 patient days, from 101.38 (95\% confidence interval (CI) 93.5 - 109.72) in the pre-implementation phase to $83.04(95 \%$ CI $74.87-91.22)$ in the post-implementation phase $(p<0.0001)$, a decline of $18.1 \%$ (95\% CI 15.71 - 20.4).

This study attests to the fact that AS can be introduced successfully into hospitals across the country that have never practised stewardship before, utilising non-infection specialists, which is especially relevant to low- and middle-income countries with limited human resources. An important question is whether the same intervention would work in the SA public sector, where the majority of the population receives healthcare. Pharmacists would need to be trained in monitoring antibiotic use, ASP project managers would need to be appointed to co-ordinate implementation and monitoring, and 'protected' stewardship time would have to be agreed upon, i.e. pharmacists (one or more) would be allowed time, according to the size of the hospital, to conduct antibiotic audit rounds. If successful, 'higher-level fruit' could be targeted, either by pharmacists alone or by pharmacists in partnership with other healthcare professionals.

Like pharmacists, nurses are a potential cadre of healthcare professionals with a proven track record in antimicrobial prescribing in SA. Primary care nurses safely initiate and re-prescribe antiretroviral therapy without detrimental effect. ${ }^{[16]}$ Furthermore, primary care nurses are at the frontline of antibiotic prescribing in the Practical Approach to Care Kit (PACK) adult intervention, a symptom-based set of guidelines for management of common conditions in primary care. ${ }^{[17]}$ Appropriate antibiotic use is written into this practical kit for nurses to direct antibiotic management. In many neighbouring countries, antibiotic stewardship has been woven into programmes such as the integrated management of childhood illness and its adolescent/adult equivalent, where once again nurses, and indeed community health workers, play a leading stewardship role. ${ }^{[18]}$ In hospitals, intensive care unit nurses would be an ideal group to take on a greater stewardship role.

Will doctors, the actual prescribers, and a group notoriously keen on safeguarding clinician prescribing autonomy, embrace pharmacists and nurses as equals in the drive towards optimising antibiotic use? The potential for pharmacist- and nurse-led models of AS to complement that of clinicians affords an exciting opportunity for SA to offer an integrated range of ASP models to serve the whole population. It will require commitment from government, hospital, clinician, pharmacist and nurse leadership to acknowledge and support their central role. It is time that we as doctors welcome other 
cadres of healthcare professionals as antibiotic stewards in their own right, able to intervene to ensure appropriate use of antibiotics and in so doing joining the fight to sustain this vital, global resource.

Acknlowledgement. We thank Angeliki P Messina, Charles Feldman, Piet J Becker, Debra A Goff, Karri A Bauer and Dilip Nathwani, co-authors of the Lancet Infectious Diseases article ${ }^{[15]}$ on which this editorial is based.

\section{Adrian J Brink}

Ampath National Laboratory Services, Milpark Hospital, Johannesburg, South Africa; and Division of Infectious Diseases and HIV Medicine, Department of Medicine, Groote Schuur Hospital and Faculty of Health Sciences, University of Cape Town, South Africa

brinka@ampath.co.za

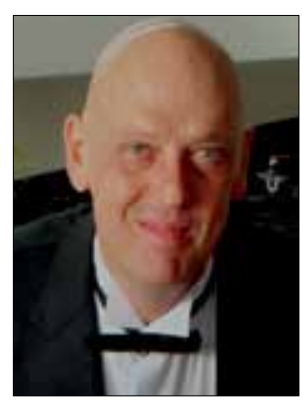

Dena van den Bergh

Department of Quality Leadership, Netcare Hospitals, Johannesburg, South Africa

\section{Marc Mendelson}

Division of Infectious Diseases and HIV Medicine, Department of Medicine, Faculty of Health Sciences, Groote Schuur Hospital, University of Cape Town, South Africa

\section{Guy A Richards}

Department of Critical Care, Charlotte Maxeke Johannesburg Academic Hospital and Faculty of Health Sciences, University of the Witwatersrand, Johannesburg, South Africa
Gelband H, Miller-Petrie M, Pant S, et al. The state of the world's antibiotics, 2015. http://cddep.org/ publications/state worlds antibiotics 2015 (accessed 21 November 2015).

2. Review on Antimicrobial Resistance. Tackling drug resistant infections globally. Final report and recommendations. http://amr-review.org/sites/default/files/160525 Final\%20paper with\%20cover. df (accessed on 14 July 2016)

3. Liu YY, Wang Y, Walsh TR, et al. Emergence of plasmid-mediated colistin resistance mechanism MCR1 in animals and human beings in China: A microbiological and molecular biological study. Lancet Infect Dis 2016;16(2):161-168. DOI:10.1016/S1473-3099(15)00424-7

4. Xavier BB, Lammens C, Ruhal R, Kumar-Singh S, Butaye P, Goossens H, Malhotra-Kumar S. Identification of a novel plasmid-mediated colistin-resistance gene, MCR-2, in Escherichia coli, Belgium, June 2016. Euro Surveill 2016;21(27):pii=30280. DOI:10.2807/1560-7917.ES.2016.21.27.30280

5. Laxminaryan R, Amábile-Cuevas C, Cars O, et al. UN high-level meeting on antimicrobials - what do we need? Lancet 2016; 388 (10041):218-220. DOI:10.1016/S0140-6736(16)31079-0

6. Brink A, Feldman C, Richards G, Moolman J, Senekal M. Emergence of extensive drug resistance (XDR) among Gram-negative bacilli in South Africa looms nearer. S Afr Med J 2008;98(8):586-590.

7. Mendelson M, Whitelaw A, Nicol M, Brink A. Wake up, South Africa! The antibiotic 'horse' has bolted. S Afr Med J 2012;102(7):607-608.

8. Coetzee J Corcoran C Prentice E et al. Emergence of plasmid-mediated colistin resistance (MCR1) among Escherichia coli isolated from South African patients. S Afr Med J 2016;106(5):449-450. DOI:10.7196/SAMJ.2016.v106i5.10710

9. National Department of Health, South Africa. Antimicrobial Resistance National Strategy Framework 2014-2024. http://www.health.gov.za/index.php/antimicrobial-resistance (accessed 14 July 2016).

10. National Department of Health, South Africa. Implementation Plan for Antimicrobial Resistance National Strategy Framework 2014-2019. http://www.health.gov.za/index.php/antimicrobial-resistance (accessed on 14 July 2016).

11. Paruk F, Richards GA, Scribante J, Bhagwanjee S, Mer M, Perrie H. Antibiotic prescription practices and their relationship to outcome in South African intensive care units. S Afr Med J 2012;102(7):613616.

12. Altiner A, Knauf A, Moebes J, Sielk M, Wilm S. Acute cough: A qualitative analysis of how GPs manage the consultation when patients explicitly or implicitly expect antibiotic prescriptions. Fam Pract 2004;21(5):500-506. DOI:10.1093/fampra/cmh505

13. Davey $\mathrm{P}$, Brown E, Charani E, et al. Interventions to improve antibiotic prescribing practices for hospital inpatients. Cochrane Database Syst Rev 2013 Apr 30;4:CD003543. DOI:10.1002/14651858. CD003543.pub3

14. Boyles, Whitelaw A, Bamford C, et al. Antibiotic stewardship ward rounds and a dedicated prescription hart reduce antibiotic consumption and pharmacy costs without affecting inpatient mortality or readmission rates. PLoS One 2013;8(12):e79747. DOI:10.1371/journal.pone.007974

15. Brink AJ, Messina AP, Feldman C, et al. on behalf of the Netcare Antimicrobial Stewardship Study Alliance. Antimicrobial stewardship across 47 South African hospitals: An implementation study. Lancet Infect Dis 2016;16(9):1017-1025. DOI:10.1016/S1473-3099(16)30012-3

16. Fairall L, Bachmann MO, Lomard C, et al. Task shifting of antiretroviral treatment from doctors to primary-care nurses in South Africa (STRETCH): A pragmatic parallel cluster-randomised trial. Lancet 2012;380(9845):889-898. DOI:10.1016/S0140-6736(12)60730-2

17. Knowledge Translation Unit PACK Western Cape, South Africa. http://knowledgetranslation.co.za/ programmes/pack-adult-wc-sa/ (accessed 14 July 2016).

18. World Health Organization. Integrated Management of Childhood Illness. http://www.who.int/ maternal_child_adolescent/topics/child/imci/en/ (accessed 14 July 2016).

S Afr Med J 2016;106(10):947-948. DOI:10.7196/SAMJ.2016.v106i10.11448 